\title{
Superposition of Emergent Monopole and Antimonopole in CoTb Thin Films
}

\author{
Yao Guang*, ${ }^{1,2}$ Kejing Ran* ${ }^{3,4}$ Junwei Zhang, ${ }^{5}$ Yizhou Liu, ${ }^{1,2}$ Senfu Zhang, ${ }^{5,6}$ Xuepeng Qiu, ${ }^{7}$ \\ Yong Peng, ${ }^{5}$ Xixiang Zhang, ${ }^{6}$ Markus Weigand, ${ }^{8}$ Joachim Gräfe, ${ }^{8}$ Gisela Schütz, ${ }^{8}$ Gerrit van \\ der Laan, ${ }^{9}$ Thorsten Hesjedal, ${ }^{10}$ Shilei Zhang, ${ }^{3,4}$ Guoqiang Yu, ${ }^{1,2,11}$ and Xiufeng Han ${ }^{1,2,11}$ \\ ${ }^{1}$ Beijing National Laboratory for Condensed Matter Physics, \\ Institute of Physics, Chinese Academy of Sciences, Beijing 100190, China \\ ${ }^{2}$ Center of Materials Science and Optoelectronics Engineering, \\ University of Chinese Academy of Sciences, Beijing 100049, China \\ ${ }^{3}$ School of Physical Science and Technology, ShanghaiTech University, Shanghai 201210, China \\ ${ }^{4}$ ShanghaiTech Laboratory for Topological Physics, \\ ShanghaiTech University, Shanghai 201210, China \\ ${ }^{5}$ Key Laboratory for Magnetism and Magnetic Materials of Ministry of Education, Lanzhou University, Lanzhou 730000, China \\ ${ }^{6}$ Physical Science and Engineering Division (PSE), \\ King Abdullah University of Science and Technology (KAUST), Thuwal 23955-6900, Saudi Arabia \\ ${ }^{7}$ Shanghai Key Laboratory of Special Artificial Microstructure Materials 85 School \\ of Physics Science and Engineering, Tongji University, Shanghai 200092, China \\ ${ }^{8}$ Max-Planck-Institut für Intelligente Systeme, Stuttgart, Germany \\ ${ }^{9}$ Magnetic Spectroscopy, Diamond Light Source, Harwell Science and Innovation Campus, Didcot OX11 0DE, United Kingdom \\ ${ }^{10}$ Department of Physics, Clarendon Laboratory, University of Oxford, Oxford OX1 3PU, United Kingdom \\ ${ }^{11}$ Songshan Lake Materials Laboratory, Dongguan, Guangdong 523808, China
}

\begin{abstract}
A three-dimensional singular point that consists of two oppositely aligned emergent monopoles is identified in continuous CoTb thin films, as confirmed by complementary techniques of resonant elastic x-ray scattering, Lorentz transmission electron microscopy, and scanning transmission x-ray microscopy. This new type of topological defect can be regarded as a superposition of an emergent magnetic monopole and an antimonopole, around which the source and drain of the magnetic flux overlap in space. We experimentally prove that the observed spin twist seen in Lorentz transmission electron microscopy reveals the cross-section of the superimposed three-dimensional structure, providing a straightforward strategy for the observation of magnetic singularities. Such a quasi particle provides an excellent platform for studying the rich physics of emergent electromagnetism.
\end{abstract}

Magnetic fields produced by multipoles are dominated by the dipole term, following the electromagnetism principle of $\nabla \cdot \mathbf{B}=0$ [1]. An elementary magnetic dipole can be constructed by a pair of a monopole (MP) and an antimonopole (AMP) that are spatially separated. MPs are regarded as fundamental particles with quantized magnetic charges [2]. The existence of a MP in real space is equivalent to that of having a local gauge transformation due to the existence of singularities in the vector potential, and a closure surface that covers a MP satisfies the $\pi_{2}\left(S^{2}\right)$ definition in the language of topology [3]. Although the theoretical arguments above do not have experimental proof so far, the MP model provides deep insights into various analogous systems in condensed matter physics. For example, an emergent MP in parameter space can effectively give rise to a Berry phase and is closely related to topological band theory [4-6].

In magnetic systems, e.g., a magnetic field applied in spin ice systems leads to the separation of emergent MPs and AMPs in real-space, producing non-trivial magnetic dynamics $[7,8]$. In the continuum limit, MPs and AMPs appear during the phase transition process between the skyrmion lattice and conical order, being responsible for unwinding topological structures [9-14]. They are also closely related to the domain wall dynamics [15-22], an aspect that is interest for spintronics. Emergent MPs thus provide a laboratory system for studying important physics such as symmetry-breaking-related dynamics due to topological defects [23], emergent electromagnetism [24], and even dynamical properties of fundamental particles [3].

The creation of various types of MP structures is the key for the exploration of MP physics. These quasi particles prefer to be spatially separated, either forming a MP-AMP dipole configuration [8], or spacing themselves out individually at different locations $[9,12,21,22,25]$. The collision of a MP-AMP pair usually results in annihilation, reminiscent of the particle-antiparticle collision process [16]. Nevertheless, MPs and AMPs may also form a bound state such as monopolonium [26, 27], which remains elusive in a magnetic system. It is therefore important to explore the existence and properties of spatially bound MP-AMP structures. From fundamental physics point of view, this simulates a scenario of how a dipole moment is formed or destroyed by infinitely proximate a MP and an AMP together [1-3]. For emergent systems, such novel texture can be regarded as a neutral MP that takes zero magnetic charge yet having non-trivial topology [24].

Another challenging task is the experimental identification of three-dimensional (3D) MP structures. The recent advances in synchrotron-based 3D magnetic imag- 


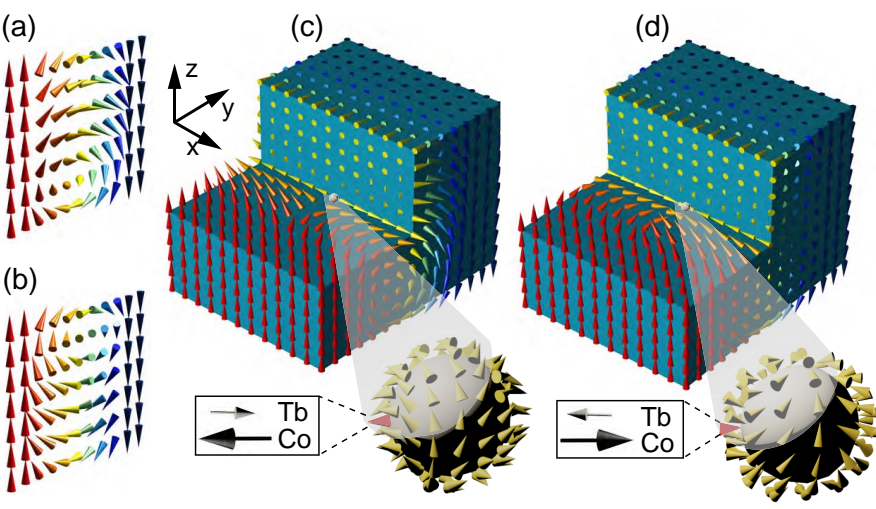

FIG. 1. Micromagnetic simulation results for a 25-nm-thick CoTb ferrimagnetic thin film. (a) Left-handed hybrid 3D domain wall with a middle layer twisting angle of $\chi=90^{\circ}$. (b) An energetically equivalent right-handed hybrid 3D domain wall structure with opposite chirality as in (a). (c,d) Two different singular points emerge when the two types of domain walls join together.

ing techniques provides powerful methods that can visualize emergent singularities [22, 25]. Nevertheless, the efficiency of such experiments is low due to the complicated data collection procedure, and the expense for obtaining a magnetic structure is high due to the nature of large-facility experiments. It is thus urgent to develop a convenient experimental strategy for the identification of three-dimensional topological spin structures.

In this Letter, we address the above two issues by creating such an emergent MP-AMP superposition in continuum CoTb thin film system, and demonstrate a simple, yet general characterization strategy for the identification of such structure. The key idea of producing such novel singular point is introduced as follows. In the continuum approximation, a thin film magnetic medium is described by the following expression for the total energy:

$$
E=\int d^{3} \mathbf{r}\left[A(\nabla \mathbf{m})^{2}+K_{u} m_{z}^{2}-\mathbf{m} \cdot \mathbf{B}\right]+E_{\text {dipolar }},
$$

where $\mathbf{m}(\mathbf{r})=\left(m_{x}, m_{y}, m_{z}\right)$ is the local magnetization, $A$ is the exchange stiffness, $K_{u}$ is the uniaxial anisotropy along $z$-axis, and $E_{\text {dipolar }}$ is the dipole-dipole interaction energy. Depending on the exact recipe of the microscopic interactions, a planar system may exhibit Bloch$\left(\chi= \pm 90^{\circ}\right)$ or Néel-type $\left(\chi=0^{\circ}, 180^{\circ}\right)$ walls, distinguished by the twisting angle $\chi[24,28]$. Recently, it was recognized that in stacked multilayer systems with alternating ferromagnetic/non-ferromagnetic units, $E_{\text {dipolar }}$ plays an important role in mediating the $2 \mathrm{D}$ domain wall structures within each layer [29-32]. Consequently, a hybrid 3D domain wall is stabilized, within which each layer can take arbitrary values of $\chi[29,30,33]$.

Figure 1(a) shows such a 3D hybrid domain wall structure with the wall direction pointing along $y$, and the thickness direction along $z$. The competing interactions described in Eq. (1) encourage a 'closure magnetization flux' pattern in the $y z$-plane. In other words, at the bottom layer $(z=0)$, a $\chi=0^{\circ}$ Néel wall is formed. Upon increasing $z$, the twisting angle increases until it reaches $90^{\circ}$ at the very middle of the stack, presenting a Blochtype wall with fixed chirality. Further increasing $z$ leads to a $\pi$-turn of the twisting angle in a left-handed rotation fashion, resulting in a $\chi=180^{\circ}$ Néel wall. Nevertheless, the chirality of the middle layer Bloch wall is not protected by the intrinsic symmetry, suggesting another degenerated, right-handed hybrid structure as shown in Fig. 1(b), in which $\chi=-90^{\circ}$ in the middle layer [32]. In fact, the hybrid 3D domain walls do not only occur in discontinuous multilayers. For a continuous magnetic thin film with finite thickness and moderate perpendicular anisotropy, our simulation suggests that similar 3D wall configurations also develop.

As the two types of degenerate 3D walls join together along the $x$-direction, emergent singularities evolve. Figure $1(\mathrm{c})$ shows the consequence of merging wall types of Fig. 1(a) and 1(b) in a tail-to-tail fashion, which commonly takes place during the domain wall formation. At the merging point, the local magnetization vectors undergo minute modifications by maintaining continuous variations in the vector field. The only exception is highlighted by the formation of a singular point in Fig. 1(c), in which its emergent magnetic field satisfies the $\pi_{2}\left(S^{2}\right)$ topological quantization for a magnetic charge $q_{m}[34,35]$ :

$$
q_{m}=\frac{1}{4 \pi} \int_{\Sigma_{k}} d s_{k} \epsilon_{i j k}\left(\partial_{i} \mathbf{n} \times \partial_{j} \mathbf{n}\right) \cdot \mathbf{n},
$$

where the local magnetization is normalized by the saturation magnetization $M_{\mathrm{S}}: \mathbf{n}=\mathbf{m} / M_{\mathrm{S}}$, and $\Sigma_{k}$ is a closure surface that covers $q_{m}$. Our numerical calculation shows that $q_{m}=1$ in this case, suggesting a magnetization configuration that resembles an emergent MP.

Next, the ferromagnetic texture above is replaced by a ferrimagnetic one [36], i.e., the local magnetization vector can be regarded as the net magnetic moment from two oppositely aligned sublattices. As shown in Fig. 1(c), if the material is ferrimagnetic, each moment that consists of a MP can be decomposed into two, labelled as $\mathrm{Tb}$ and Co moments in this case. Consequently, the singularity resembles the MP-AMP superposition structure, i.e, the source of emergent field produced by the Co overlaps with the drain of emergent field produced by $\mathrm{Tb}$. On the other hand, if the two types of 3D hybrid domain walls are joined in a head-to-head fashion, a similar superposition singular point with a Co-based AMP and an Tb-based MP is formed, as shown in Fig. 1(d).

Following by the above concept, ferrimagnetic $\mathrm{CoTb}$ thin films were synthesized by magnetron sputtering [35]. All characterizations were carried out in zero field on a 25-nm-thick CoTb thin film, capped with $5 \mathrm{~nm}$ Pt. Figures 2(a) and 2(b) show typical scanning transmission 

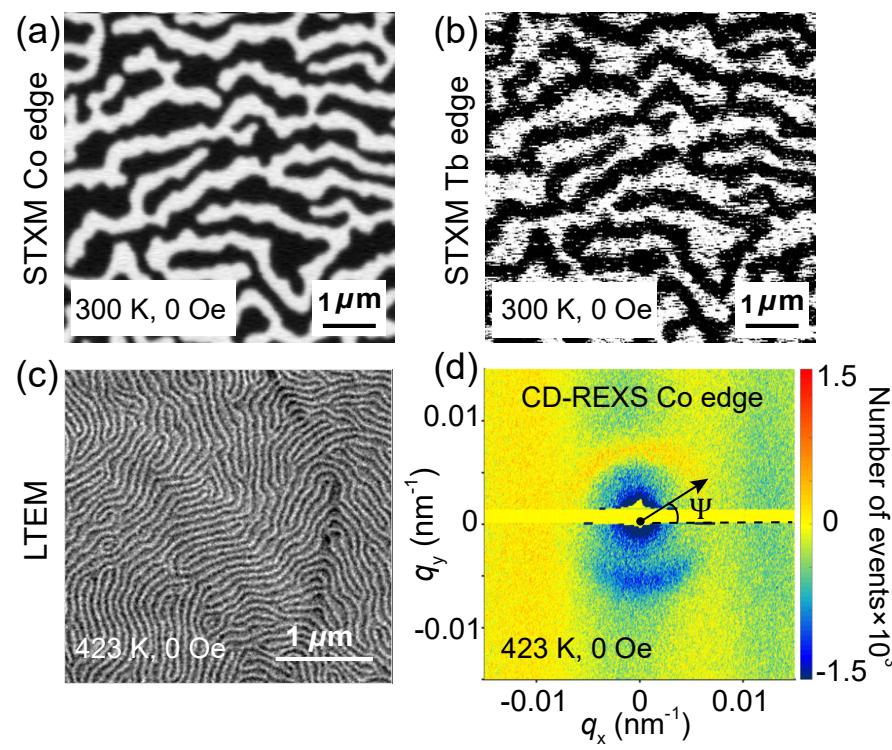

FIG. 2. (a,b) STXM images for 25-nm-thick CoTb film measured at the Co and $\mathrm{Tb}$ edges, respectively. (c) LTEM image of the 25 -nm-thick CoTb film measured at $423 \mathrm{~K}$. (d) CD-REXS pattern measured for the same sample and same condition as in (c).

x-ray microscopy (STXM) images for the same CoTb sample, targeted near the Co $L_{3}(779 \mathrm{eV})$ and $\mathrm{Tb} M_{5}$ edges $(1238 \mathrm{eV})$, respectively. STXM images show $m_{z}$ configuration of the domain wall pattern with element specification. The labyrinth domain can be clearly identified. The opposite circular dichroism (CD) signals for the $\mathrm{Co}$ and $\mathrm{Tb}$ edges provides direct evidence that the $\mathrm{Co}$ and $\mathrm{Tb}$ moments are antiparallel, a signature of ferrimagnetism [36].

In order to characterize the in-plane properties $\left(m_{x}-m_{y}\right.$ components) of the magnetic structure, Lorentz transmission microscopy (LTEM) measurements were performed on an identical film at an elevated temperature of $423 \mathrm{~K}$. At higher temperature, the modulation periodicity $\lambda_{h}$ decreases, while the detailed domain wall structure remains the same. This leads to an increased scattering wavevector $q_{h}=1 / \lambda_{h}$ in reciprocal space, which simplifies the successive REXS measurements. Figure 2(c) shows a typical LTEM image measured at zero-tilt angle. A similar labyrinth domain pattern can be clearly identified, which is consistent with the STXM results. For typical LTEM analysis, the specimen presents Bloch-type modulations if the magnetic signal is observable under zero-titling [37]. On the other hand, the smearing-out of the magnetic signal at zero oblique angle indicates a Néel-type wall structure [31, 38, 39]. Therefore, the existence of clear magnetic domain contrast in Fig. 2(c) leads to the straightforward conclusion that CoTb hosts Bloch-type domain wall.

Next, we performed resonant elastic x-ray scattering (REXS) measurements in order to retrieve the exact $\chi$ value. It has been established that CD-REXS is the ideal technique for probing the twisting angle of modulated magnetization domain walls $[28,30,40]$. The direct CDREXS data is represented as a half-positive-half-negative diffraction pattern by CD intensity around a circle of fixed radius. The radius of the circle is associated with the domain wall propagating wavevector $q_{h}$, and the azimuthal angle $\Psi$ is used to define the angular position on the ring. Thus, the CD-REXS pattern around the circle $I_{\mathrm{CD}}(\Psi)$ unambiguously reveals the twisting angle : $I_{\mathrm{CD}}(\Psi) \propto \sin (\Psi+\chi)[28,40]$.

Figure 2(d) shows the CD-REXS pattern measured on the same CoTb structure as that for LTEM. The half-redhalf-blue configuration with a horizontal dividing line directly yields $\chi=0^{\circ}$, leading to the conclusion that $\mathrm{CoTb}$ is governed by a Néel-type domain wall. This strongly contradicts the LTEM results, suggesting more subtle underlying physics $[28,30,40]$.

In order to address the experimental discrepancy, one has to consider rather prudent interpretation of LTEM data. Indeed, LTEM measures the average magnetic signal from all layers along $z[31,32,37]$. Therefore, for a magnetic thin film sample of finite thickness, the depth-dependent information is lost in the LTEM contrast. From this perspective, we argue that whether LTEM shows magnetic contrast or not depends on the exact $\chi(z)$ profile, and not directly related to the simple $2 \mathrm{D}$ wall type. If the $\chi(z)$ profile contains most of the layers with Néel-type walls and ignorable Bloch components, the magnetic signals will smear out. On the other hand, if the $\chi(z)$ profile contains most of the layers that present Bloch-component, observable magnetic domain contrast will appear, as if the system presents pure Bloch-type domain walls. In our case, the topmost and bottommost layers are invisible to magnetic imaging, while the middle layer contributes to discernible magnetic signals as shown in Fig. 2(c).

Meanwhile, REXS probes the same system in a completely different manner due to the reflection geometry. The x-ray penetration condition suggests that the measured $I_{\mathrm{CD}}^{\text {total }}$ takes the form of $I_{\mathrm{CD}}^{\text {total }}=\sum_{z} I_{\mathrm{CD}}(z) e^{-z / L_{z}}$, where $L_{z}$ describes the penetration depth that is a function of the x-ray incidence angle and the photon energy $[40,41]$. This means that the topmost layers dominate more in $I_{\mathrm{CD}}^{\text {total }}$ compared to the layers buried underneath. This explains why the CD-REXS signal [Fig. 2(d)] shows $\chi=0^{\circ}$ even for a Néel-Bloch-Néel 3D configuration. Therefore, the apparent contradiction between LTEM and CD-REXS can be resolved by assuming ferrimagnetic CoTb thin films to host 3D hybrid domain walls, a crucial precondition towards the realization of emergent charge-neutral monopoles.

In order to provide unambiguous experimental evidence for the existence of hybrid 3D domain walls in our film, a depth-dependent study that reveals the domain wall type at each layer is required. The depth-dependent 


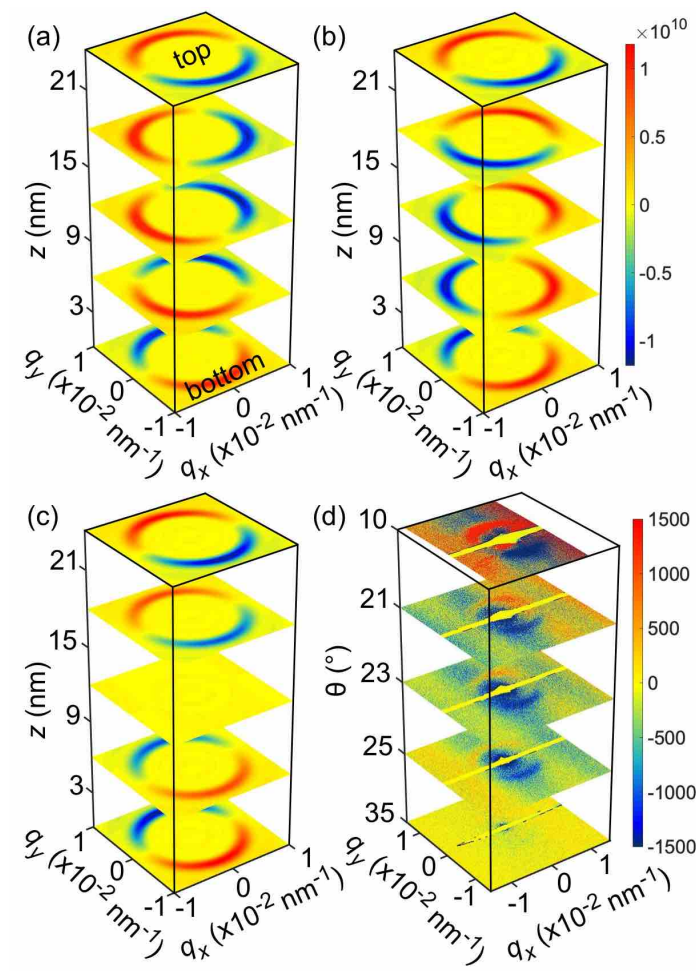

FIG. 3. (a) Calculated layer-specific CD-REXS pattern $I_{\mathrm{CD}}(z)$ for a left-handed hybrid wall, and (b) for a righthanded hybrid wall. (c) Calculated layer-specific CD-REXS pattern for the coexistence of left- and right-handed hybrid walls. (d) Angular dependence of the experimentally measured $I_{\mathrm{CD}}^{\mathrm{total}}$.

$\chi(z)$ information can be obtained by varying the x-ray incidence angle $\theta[29,30]$. At a particular $\theta, I_{\mathrm{CD}}^{\text {total }}$ encodes the $\chi(z)$ information from all layers with weighted intensities. By varying $\theta$, the weighting configuration changes, leading to a different $I_{\mathrm{CD}}^{\text {total }}$ pattern. The analysis of the measured $I_{\mathrm{CD}}^{\text {total }}$ signal as a function of $\theta$ allows one to gain knowledge of $I_{\mathrm{CD}}(z)$, therefore the information about $\chi(z)$.

Figures 3(a) and 3(b) show the calculated layer-specific CD-REXS pattern for left- and right-handed hybrid walls, respectively, i.e, the $I_{\mathrm{CD}}(z)$ pattern. It is clear that $I_{\mathrm{CD}}(z)$ follows an identical evolution as that of the $\chi(z)$ profile. At the very top $\left(\chi=180^{\circ}\right)$ and very bottom $\left(\chi=0^{\circ}\right)$ layer, both left and right hybrid walls have identical CD-REXS patterns. While approaching the middle layer, the CD-REXS pattern starts to twist with different rotation sense. For a left-handed wall, the CD pattern rotates in a counterclockwise fashion from the top surface to the bottom, as shown in Fig. 1(a); while the right-handed wall behaves oppositely. If both chiral structures coexist with the same density, the total layer-specific $I_{\mathrm{CD}}(z)$ will take the average of both. Figure 3(c) shows the calculated CD-REXS pattern as a function of $z$ for a system that hosts both chiral hybrid domain walls. Interestingly, the dividing vector that separates positive and negative regions of the CD signals does not rotate at all. The CD-REXS patterns for the upper layers are uniform with decreasing CD intensities while approaching the middle, and the bottom-half part has a sudden CD sign reversal. At the very middle layer, the CD signals are completely cancelling each other, giving zero intensity.

Figure $3(\mathrm{~d})$ shows the measured $I_{\mathrm{CD}}^{\text {total }}$ patterns at different $\theta$. At the smallest possible angle of $10^{\circ}$, a clear CD-REXS ring with horizontal dividing vector can be identified. At elevated $\theta$, the red-blue ring keeps its configuration, until it completely vanishes at $\theta=35^{\circ}$, the largest angle possible in our experimental setup. It is worth noting that for the CoTb system at the Co $L_{3}$ resonance condition, the largest $L_{z}$ is merely $12 \mathrm{~nm}$. Consequently, the bottom-half layers that are responsible for the CD sign reversal do not contribute sufficiently to the overall measured $I_{\mathrm{CD}}^{\text {total }}$. Therefore, at $\theta=35^{\circ}$, the CDsign-reversal cannot be observed. Instead, an extinction of the CD-REXS pattern appears. Thus, the observed angular dependent CD-REXS is well in agreement with the layer-specific $I_{\mathrm{CD}}(z)$ profile shown in Fig. 3(c). This directly proves that both types of hybrid $3 \mathrm{D}$ domain walls [Fig. 1(a) and 1(b)] coexist in our ferrimagnetic thin films. Therefore, all conditions that are needed for creating emergent MP-AMP superposition singularities are met in our system.

It is therefore straightforward to look for such structures in the microscopy images, i.e., where left- and righthanded hybrid domain walls join together. We have established that for LTEM, the measured magnetic contrast is predominantly coming from the very middle layer of the film at $z=z_{m}$. In other words, the singular points in the LTEM images are direct cross-sectional cuts of the $3 \mathrm{D}$ topological volume structure. This provides us a convenient method for observing the MP-AMP superposition singularities via transport-of-intensity (TIE) reconstruction [37].

Figure 4(a) shows typical LTEM images at $300 \mathrm{~K}$ and 0 Oe, specifically measuring the Bloch component of the domain wall around the middle layer of the $3 \mathrm{D}$ film. The Bloch-components of the 3D domain walls exhibit a black-to-white contrast transitions across the worm-like lines. Along the same 'worm lines', defect points can be found along which the wall order is reversed by whiteto-black fashion, as marked by the yellow squares in Fig. 4(a). This is direct evidence that a left-handed hybrid wall with $\chi\left(z_{m}\right)=90^{\circ}$ joins together with a right-handed one with $\chi\left(z_{m}\right)=-90^{\circ}$. The emergent MP-AMP pair is therefore tied to the merging point. In order to visualize the net magnetization flux lines of the topological defect, TIE was performed at the marked region. Figure 4(b) shows the detailed internal structure of a tail-totail singular point, which is well in agreement with the theoretical predictions as shown in Fig. 1(c). On the 


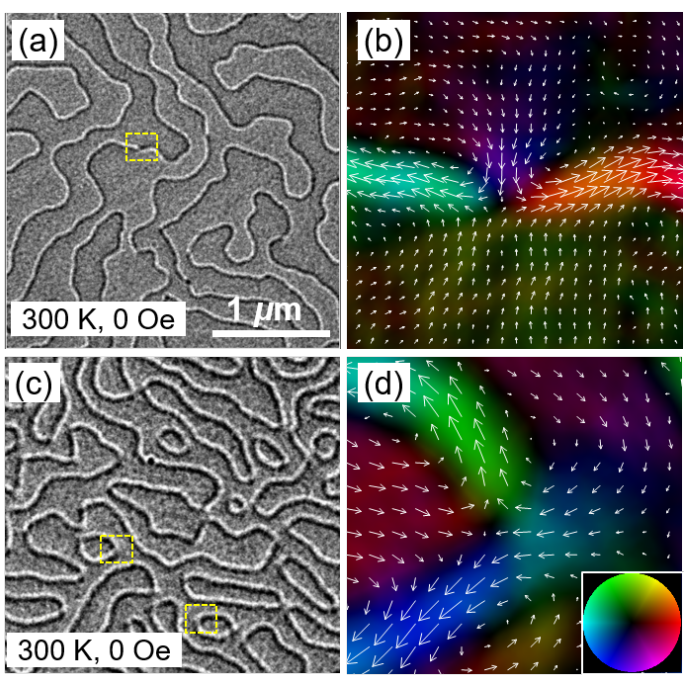

FIG. 4. (a) LTEM images for 25-nm-thick CoTb film measured at $300 \mathrm{~K}$, from which tail-to-tail MP-AMP superposition point is found and marked by the yellow square. (b) TIE reconstruction of the yellow square region in (a). (c,d) same as in $(\mathrm{a}, \mathrm{b})$, showing head-to-head MP-AMP pairs.

other hand, Figs. 4(c) and 4(d) show another MP-AMP pair with head-to-head configuration, which is consistent with the texture shown in Fig. 1(d). Therefore, we have successfully created and observed such novel topological structure.

In summary, the spatially overlapping emergent MPAMP singular point can be nucleated in ferrimagnetic CoTb thin films. Such a complex defect represents a novel topological structure that is interesting for studying both fundamental and emergent electromagnetism. Most importantly, 3D hybrid domain walls are commonly found in continuous magnetic thin films with finite thickness and moderate perpendicular magnetic anisotropy. Therefore, LTEM measurements need to be interpreted by taking into account the exact $\chi(z)$ profile. In fact, most of the LTEM contrast probes the particular layers where Bloch-components dominate. This provides an experimentally convenient strategy for visualizing cross-sectional cuts of the $3 \mathrm{D}$ singularities in general.

Acknowledgments This work was supported by the National Key Research and Development Program of China (Grant No. 2017YFA0206200), the National Natural Science Foundation of China (Grant No.11874409, 51831012), the Beijing Natural Science Foundation (Grant No. Z190009), the Science Center of the National Science Foundation of China (Grant No. 52088101), and the K. C. Wong Education Foundation (Grant No. GJTD-2019-14). S.L.Z. acknowledges the National Key R \& D Program of China under contract number 2020YFA0309400, and the National Natural Science
Foundation of China (Grant no.12074257). X.X.Z acknowledges the support from King Abdullah University of Science and Technology with funding numbers: ORS2019-CRG8-4081. T.H. acknowledges support from the Engineering and Physical Science Research Council (UK) under grant EP/N032128/1. K.J.R. acknowledges the support from the Shanghai Sailing Program (Grant No. 20YF1430600) and the National Natural Science Foundation of China (Grant No. 12004249). Diamond Light Source is acknowledged for beamtime on I10 under proposal number MM-23785.

*These authors contributed equally to the work.
zhangshl1@shanghaitech.edu.cn
guoqiangyu@iphy.ac.cn

[1] K. A. Milton, Rep. Prog. Phys. 69, 1637 (2006).

[2] P. A. M. Dirac, Proc. Roy. Soc. (London) A 133, 60 (1931).

[3] A. Vilenkin and E. P. S. Shellard, Cosmic Strings and Other Topological Defects (Cambridge Univ. Press, 1994).

[4] Z. Fang, N. Nagaosa, K. S. Takahashi, A. Asamitsu, R. Mathieu, T. Ogasawara, H. Yamada, M. Kawasaki, Y. Tokura, and K. Terakura, Science 302, 92 (2003).

[5] D. Xiao, M. C. Chang, and Q. Niu, Rev. Mod. Phys. 82, 1959 (2010).

[6] X. L. Qi and S. C. Zhang, Rev. Mod. Phys. 83, 1057 (2011).

[7] C. Castelnovo, R. Moessner, and S. L. Sondhi, Nature 451, 42 (2008).

[8] D. J. P. Morris, D. A. Tennant, S. A. Grigera, B. Klemke, C. Castelnovo, R. Moessner, C. Czternasty, M. Meissner, K. C. Rule, J. U. Hoffmann, K. Kiefer, S. Gerischer, D. Slobinsky, and R. S. Perry, Science 326, 411 (2009).

[9] P. Milde, D. Köhler, J. Seidel, L. M. Eng, A. Bauer, A. Chacon, J. Kindervater, S. Mühlbauer, C. Pfleiderer, S. Buhrandt, C. Schütte, and A. Rosch, Science 340, 1076 (2013).

[10] F. N. Rybakov, A. B. Borisov, S. Blügel, and N. S. Kiselev, Phys. Rev. Lett. 115, 117201 (2015).

[11] J. Wild, T. N. G. Meier, S. Pöllath, M. Kronseder, A. Bauer, A. Chacon, M. Halder, M. Schowalter, A. Rosenauer, J. Zweck, J. Müller, A. Rosch, C. Pfleiderer, and C. H. Back, Sci. Adv. 3, e1701704 (2017).

[12] F. Zheng, F. N. Rybakov, A. B. Borisov, D. Song, S. Wang, Z.-A. Li, H. Du, N. S. Kiselev, J. Caron, A. Kovács, M. Tian, Y. Zhang, S. Blügel, and R. E. Dunin-Borkowski, Nat. Nanotech. (2018).

[13] N. Kanazawa, A. Kitaori, J. S. White, V. Ukleev, H. M. Rønnow, A. Tsukazaki, M. Ichikawa, M. Kawasaki, and Y. Tokura, Phys. Rev. Lett. 125, 137202 (2020).

[14] K. Ran, Y. Liu, Y. Guang, D. M. Burn, G. van der Laan, T. Hesjedal, H. Du, G. Yu, and S. Zhang, Phys. Rev. Lett. 126, 017204 (2021).

[15] Y. P. Kabanov, L. M. Dedukh, and V. I. Nikitenko, JETP Lett. 49, 637 (1989).

[16] R. Hertel and C. M. Schneider, Phys. Rev. Lett. 97, 
$177202(2006)$

[17] A. Thiaville and Y. Nakatani, Spin Dynamics in Confined Magnetic Structures III (Springer, Berlin, 2006).

[18] H. G. Piao, J. H. Shim, D. Djuhana, and D. H. Kim, Appl. Phys. Lett. 102, 112405 (2013).

[19] S. D. Col, S. Jamet, N. Rougemaille, A. Locatelli, T. O. Mentes, B. S. Burgos, R. Afid, M. Darques, L. Cagnon, J. C. Toussaint, and O. Fruchart, Phys. Rev. B 89, 180405(R) (2014).

[20] P. Wohlhüter, M. T. Bryan, P. Warnicke, S. Gliga, S. E. Stevenson, G. Heldt, L. Saharan, A. K. Suszka, C. Moutafis, R. V. Chopdekar, J. Raabe, T. Thomson, G. Hrkac, and L. J. Heyderman, Nat. Commun. 6, 7836 (2015).

[21] M. Y. Im, H. S. Han, M. S. Jung, Y. S. Yu, S. Lee, S. Yoon, W. Chao, P. Fischer, J. Hong, and K. S. Lee, Nat. Commun. 10, 593 (2019).

[22] A. Hierro-Rodriguez, C. Quirós, A. Sorrentino, L. M. Alvarez-Prado, J. I. Martín, J. M. Alameda, S. McVitie, E. Pereiro, M. Vélez, and S. Ferrer, Nat. Commun. 11, $6382(2020)$.

[23] N. D. Mermin, Rev. Mod. Phys. 51, 591 (1979).

[24] N. Nagaosa and Y. Tokura, Nat. Nanotech. 8, 899 (2013).

[25] C. Donnelly, M. Guizar-Sicairos, V. Scagnoli, S. Gliga, M. Holler, J. Raabe, and L. J. Heyderman, Nature 547, 328 (2017).

[26] C. T. Hill, Nuclear Physics B 224, 469 (1983).

[27] L. Epele, H. Fanchiotti, C. García Canal, and V. Vento, The European Physical Journal C 56, 87 (2008).

[28] S. L. Zhang, G. van der Laan, and T. Hesjedal, Phys. Rev. B 96, 094401 (2017).

[29] W. Legrand, J.-Y. Chauleau, D. Maccariello, N. Reyren, S. Collin, K. Bouzehouane, N. Jaouen, V. Cros, and A. Fert, Sci. Adv. 4 (2018).

[30] W. Li, I. Bykova, S. Zhang, G. Yu, R. Tomasello, M. Carpentieri, Y. Liu, Y. Guang, J. Gräfe, M. Weigand, D. M.
Burn, G. van der Laan, T. Hesjedal, Z. Yan, J. Feng, C. Wan, J. Wei, X. Wang, X. Zhang, H. Xu, C. Guo, H. Wei, G. Finocchio, X. Han, and G. Schütz, Advanced Materials 31, 1807683 (2019).

[31] J. A. Garlow, S. D. Pollard, M. Beleggia, T. Dutta, H. Yang, and Y. Zhu, Phys. Rev. Lett. 122, 237201 (2019).

[32] S. D. Pollard, J. A. Garlow, K.-W. Kim, S. Cheng, K. Cai, Y. Zhu, and H. Yang, Phys. Rev. Lett. 125, 227203 (2020).

[33] Y. Dovzhenko, F. Casola, S. Schlotter, T. X. Zhou, F. Büttner, R. L. Walsworth, G. S. D. Beach, and A. Yacoby, Nat. Commun. 9, 2712 (2018).

[34] G. E. Volovik, Journal of Physics C: Solid State Physics 20, L83 (1987).

[35] See Supplemental Material for details.

[36] S. Woo, K. M. Song, X. C. Zhang, Y. Zhou, M. Ezawa, X. X. Liu, S. Finizio, J. Raabe, N. J. Lee, S. Kim, S. Y. Park, Y. Kim, J. Y. Kim, D. Lee, O. Lee, J. W. Choi, B. C. Min, H. C. Koo, and J. Chang, Nat. Commun. 9, 959 (2018).

[37] X. Z. Yu, Y. Onose, N. Kanazawa, J. H. Park, J. H. Han, Y. Matsui, N. Nagaosa, and Y. Tokura, Nature 465, 901 (2010).

[38] M. J. Benitez, A. Hrabec, A. P. Mihai, T. A. Moore, G. Burnell, D. McGrouther, C. H. Marrows, and S. McVitie, Nat. Commun. 6, 8957 (2015).

[39] S. D. Pollard, J. A. Garlow, J. Yu, Z. Wang, Y. Zhu, and H. Yang, Nat. Commun. 8, 14761 (2017).

[40] S. Zhang, G. van der Laan, W. Wang, A. Haghighirad, and T. Hesjedal, Phys. Rev. Lett. 120, 227202 (2018).

[41] S. Zhang, G. van der Laan, J. Müller, L. Heinen, M. Garst, A. Bauer, H. Berger, C. Pfleiderer, and T. Hesjedal, Proc. Natl. Acad. Sci. U.S.A. 115, 6386 (2018) 